\title{
Health Care Needs of Women Aged 40 and Over Attending an Inner City Integrated Sexual Health Clinic
}

\section{Patrice Grech, Rebecca Marchant, Mannampallil Samuel \\ King's College Hospital NHS Foundation Trust, London}

\section{Background}

- Sexual health policy is targeted towards younger adults, with national screening programmes and research studies excluding individuals over the age of 44.

- However there is evidence to suggest that up to $80 \%$ of individuals continue to be sexually active in later life ${ }^{1}$.

- A study found that $7 \%$ of older people engaged in sexual practices that could put them at risk of contracting a sexually transmitted infection $(\mathrm{STI})^{1}$.

- This suggests that sexual risk taking behaviour is not limited to the younger population. Indeed, UK surveillance data demonstrated that rates of STIs doubled in older people between 1996 and 2003, the fastest rise in all age groups ${ }^{2}$.
Aim

- To assess the health care needs of women aged 40 and over attending an integrated sexual health clinic in South London.

\section{Method}

- Retrospective case note review of 200 randomly selected female patients aged 40 and over attending clinic between 2nd June 2014 and 30th May 2015

- Data collected included demographics, reason for attendance, STIs diagnosed and use of condoms/other contraception.

Results

\begin{tabular}{ll} 
& Demographics \\
\hline Ethnicity & $\%$ \\
\hline Black & 55 \\
White & 29 \\
Other & 16 \\
Sexuality & $\%$ \\
\hline Heterosexual & 81.5 \\
Homosexual & 0.5 \\
Bisexual & 1 \\
Not specified & 17 \\
\hline
\end{tabular}

- $1728 / 5039$ women (34\%) were aged 40 and over. Mean age was 46.6 years (range: $40-73$ years).

- 3 women were pregnant at time of visit. $170 / 200$ notes documented whether there was a history of termination of pregnancy (TOP) or not; 60 women (35\%) previously had TOP. $43.3 \%$ of these underwent $>1$ TOP.

- $47.5 \%$ of patients were symptomatic at presentation (Fig. 1). Some attended for more than 1 reason. The presenting symptoms are categorised in Fig. 2.

- 150/200 women received testing (of any type) and/or clinical examination; 29 (19.3\%) were found to have an STI.

- Other (non-STI such as Candida, Bacterial vaginosis and urinary tract infection) diagnoses were made in 57/200 (28.5\%).

- Documentation of whether condoms were used or not was found in $170 / 200$ notes. Of those who had this documentation: 38 were using condoms (22.4\%), 128 were not using condoms $(75.3 \%)$ and 4 women were not sexually active (2.4\%).

- $106 / 200(53 \%)$ overall were using some method of contraception.

\section{Fig 1. Reason for Clinic Attendance}

\section{Fig 2. Presenting Symptoms}

\section{0}

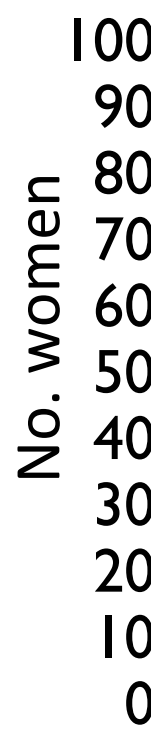
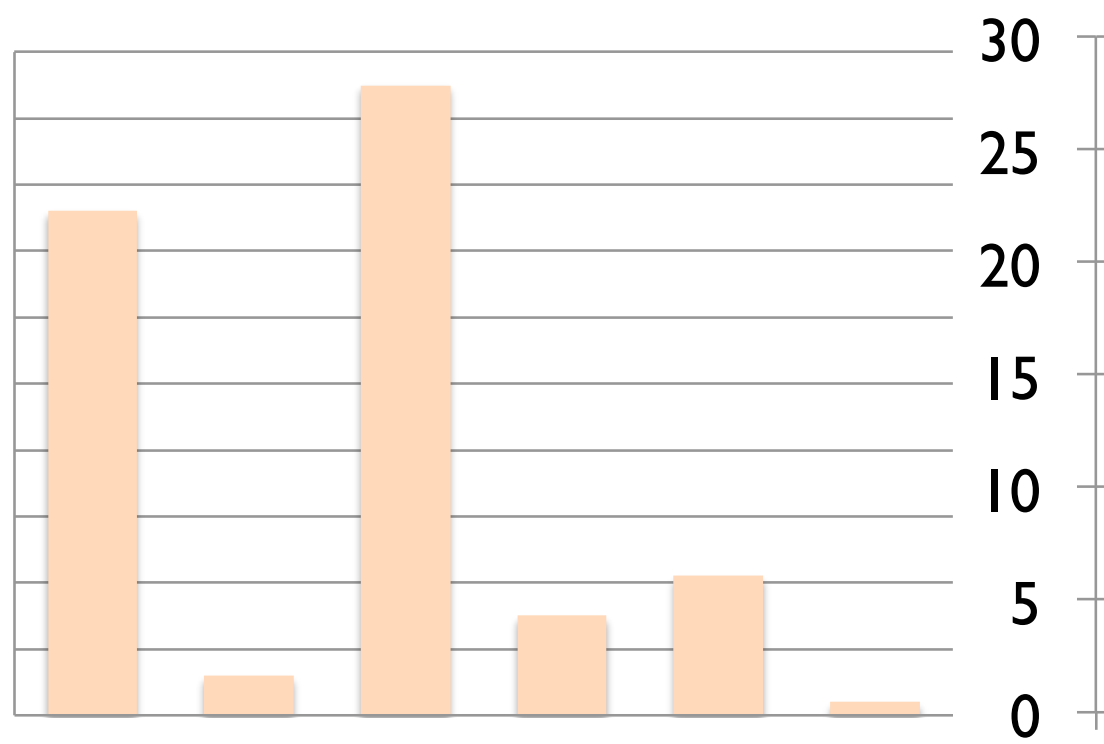

0

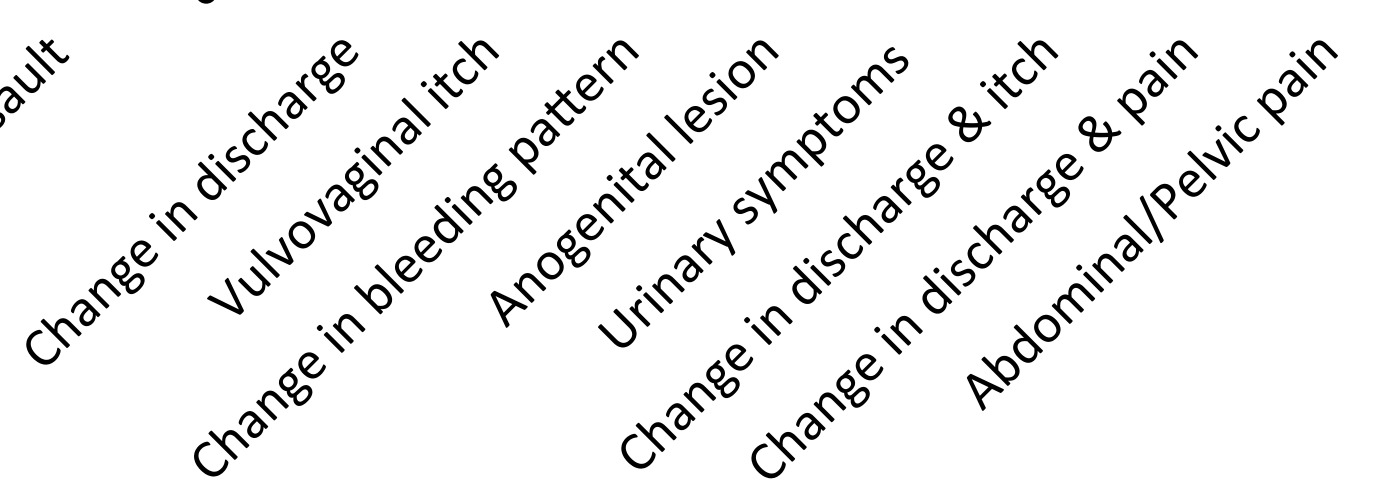

*Other STI-related refers to partner notification, symptoms in a recent sexual partner or returning for treatment/follow-up.

**AARC - long-acting reversible contraception

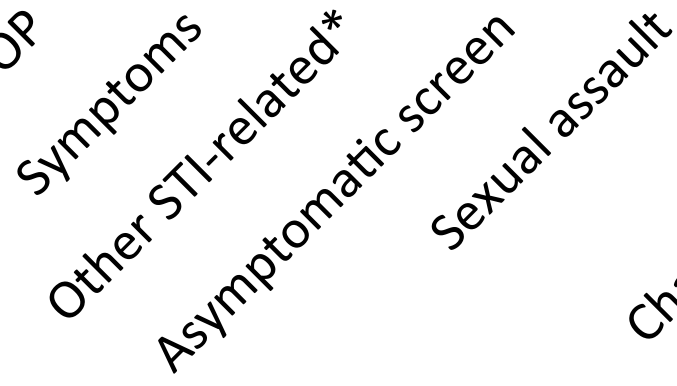

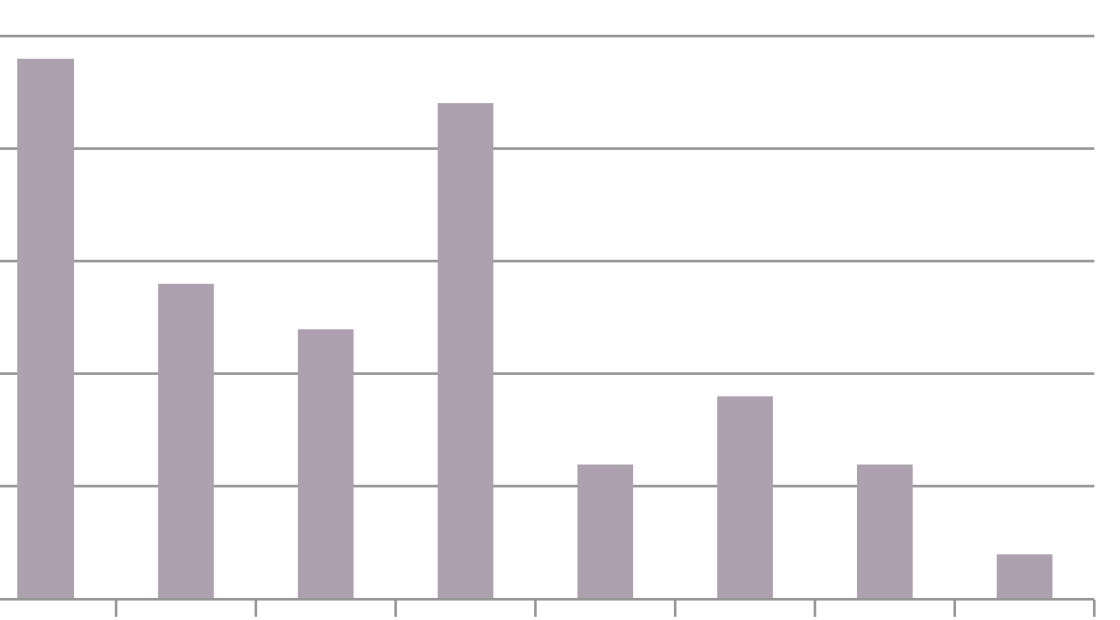

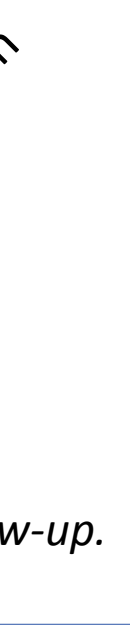

\begin{tabular}{lrr}
\multicolumn{2}{c}{ STI Diagnoses } \\
\hline STI & $\mathbf{n}$ & $\mathbf{\%}$ \\
\hline Genital Herpes & 8 & 5.3 \\
Chlamydia & 2 & 1.3 \\
Gonorrhea & 1 & 0.7 \\
Trichomonas Vaginalis & 7 & 4.7 \\
Syphillis & 3 & 2.0 \\
Anogenital Warts & 5 & 3.3 \\
Pelvic Inflammatory Disease & 2 & 1.3 \\
Hepatitis B & 1 & 0.7 \\
\hline
\end{tabular}

\section{Contraceptive Requests}

Type of contraception requested $\mathrm{n}$

Emergency contraception 4.3

LARC** insertion

LARC** removal

$36 \quad 47.4$

Coil check

Oral contraceptive pill

Advice only

Total

\section{Discussion}

- A significant proportion of patients accessing GUM services were over the age of 40, many of whom were symptomatic.

- STI rates were high in this group.

- More than one third of women had a history of TOP and condom use was low overall at $22.4 \%$.

- These findings may indicate sexual risk taking behaviour in older women.

- The sexual health needs of older people can only continue to rise, given our rapidly ageing population.

- It is therefore necessary to challenge assumptions regarding sexuality in older age groups and develop health promotion strategies specific to them. 